01

\title{
Возбуждение ридберговских атомов таллия в электрическом поле
}

\author{
(ㄱ П.А. Бохан ${ }^{1}$, Дм.Э. Закревский ${ }^{1}$, В.А. Ким ${ }^{1}$, Н.В. Фатеев ${ }^{1,2, \text { Ф }}$ \\ ${ }^{1}$ Институт фоизики полупроводников им. А.В. Ржанова СО РАН, \\ 630090 Новосибирск, Россия \\ ${ }^{2}$ Новосибирский государственный университет, \\ 630090 Новосибирск, Россия \\ ฯ e-mail: fateev@isp.nsc.ru
}

Поступила в редакцию 05.05.2017 г.

Исследован спектр возбуждения ридберговских состояний атомов таллия с использованием коллимированного атомного пучка в двухступенчатой изотопически селективной лазерной схеме $6^{2} P_{1 / 2} \rightarrow 6^{2} D_{3 / 2} \rightarrow \mathrm{Tl}^{* *}$ в присутствии электрического поля с напряженностью до $1.5 \mathrm{kV} / \mathrm{cm}$ вблизи уровня $16 F_{5 / 2}$. Экспериментально изучены индуцированные внешним электрическим полем дипольно запрещенные оптические переходы $6 D_{3 / 2} \rightarrow 18 D_{3 / 2}$ и $6 D_{3 / 2} \rightarrow 16 G_{7 / 2}$. Получены значения для скалярных поляризуемостей (в ед. $\left.\mathrm{cm}^{-1} /(\mathrm{kV} / \mathrm{cm})^{2}\right): \alpha_{0}\left(16 F_{5 / 2}\right)=3.71 \pm 0.3, \quad \alpha_{0}\left(18 D_{3 / 2}\right)=11.70 \pm 0.25 \quad$ и $\alpha_{0}\left(16 G_{7 / 2}\right)=44.1 \pm 0.9$, которые сравниваются с расчетными. Определены новые значения энергетических параметров для состояний $18 D_{3 / 2}$ и $16 G_{7 / 2}$.

DOI: $10.21883 /$ OS.2018.01.45349.57-17

\section{Введение}

Количественные измерения энергетических сдвигов в атомах, вызванных присутствием внешнего электрического поля, являются необходимым при проверке точности теоретических методов, направленных на расчет атомных волновых функций в многоэлектронных атомных системах. Эти расчеты особенно актуальны для атома таллия, принадлежащего III группе периодической системы. Таллий является важной тест-системой для проверки нарушения дискретной симметрии [1]. Результаты для таллия могут быть применены и к другим трехвалентным атомам, таким как алюминий, галлий и индий. Исследования эффекта Штарка представляют большой интерес из-за его применения в разработке нового поколения оптических атомных часов, оптических систем охлаждения, изучении дальнодействующих сил между атомными частицами и т.д. [2]. Эти исследования могут предоставить информацию об электрическом дипольном моменте электронов [3] и несохранении четности в атомах [4]. Теоретические расчеты электрических дипольных поляризуемостей для некоторых атомов достигли значительного развития за последние годы [5].

Особую группу составляют исследования с ридберговскими атомами, поскольку в этом случае значение поляризуемости атомов в электрическом поле растет пропорционально седьмой степени номера главного квантового числа. Точные экспериментальные данные для сильно возбужденных состояний обеспечивают дальнейшую проверку теоретических расчетов. Большое количество экспериментов по штарковской спектроскопии проведено для щелочных и щелочно-земельных атомов [6]. Однако значительно реже сообщается об экспериментальных результатах для поляризуемости сложных атомов. Это связано с трудностями экспериментальной реализации спектроскопии высокого разрешения высоковозбужденных состояний.

Атом таллия является одним из самых тяжелых элементов III группы, имеющим два стабильных изотопа ${ }^{203} \mathrm{Tl}$ и ${ }^{205} \mathrm{Tl}$, с относительным содержанием в природной смеси соответственно 29.5 и $70.5 \%$ со спином $1 / 2$. С теоретической точки зрения атом таллия имеет простую электронную структуру с одним валентным p-электроном вне замкнутых оболочек, спектры которого структурированы как у водородоподобных атомов. Однако простая структура типа $n s^{2} n l$ в $\mathrm{Tl}$ возмущается существованием более сложных конфигураций, таких как $n s n p^{2}$. Взаимодействие этих двух конфигураций обладает сильным влиянием на различные атомные параметры (интервалы тонкой структуры, силы осцилляторов электрических дипольных переходов и др.). В результате сложного строения ядра квантовые дефекты энергетических состояний велики. Это приводит к сложной картине поведения спектров оптических переходов в присутствии электрического поля. Вследствие этого штарковская структура энергетических уровней таллия оказывается не подобной спектрам щелочных атомов. Недавние расчеты эффекта Штарка для атома галлия, принадлежащего также III группе элементов, показали [7], что его штарковская структура уровней отличается от структуры ранее широко исследованных атомов щелочных металлов.

В настоящей работе впервые сообщаются экспериментальные результаты по штарковской спектроскопии ридберговских состояний атомов таллия в окрестности уровня $16^{2} F_{5 / 2}$ с использованием излучения узкополосных перестраиваемых по частоте лазеров и коллимированного атомного пучка. Целью экспериментальных исследований являлось получение абсолютных значений скалярных поляризуемостей энергетических состояний 
и их сравнение с расчетными. Параллельно регистрировались запрещенные переходы, которые проявляются изза перемешивания энергетических уровней с различными значениями орбитального момента $L$ в присутствии внешнего электрического поля. Этот факт известен уже давно, однако для сложных атомов он теоретически и экспериментально мало исследован. Такие переходы, индуцированные электрическим полем для атомов III группы, ранее исследовались только для атомов алюминия [8]. Этот процесс представляет интерес для лазерного разделения изотопов таллия. В этом случае появляется возможность использования узкополосных лазеров с фиксированной частотой излучения для эффективного возбуждения ридберговских состояний. При этом точная настройка линии поглощения нужного изотопа на частоту излучения лазера может производиться с помощью изменения напряженности внешнего электрического поля. Это приводит к значительному упрощению маршрута селективного возбуждения атомов для лазерного разделения изотопов.

\section{Методика эксперимента}

Полное описание экспериментальной установки и методики измерений содержится в работах [9-11]. Здесь приводятся только основные детали, относящиеся к исследованиям, выполненным в этой работе. Возбуждение атомов $\mathrm{Tl}$ на ридберговские состояния осуществлялось по двухступенчатой схеме $6 P_{1 / 2} \rightarrow 6 D_{3 / 2} \rightarrow n F_{5 / 2}$. На первой ступени использовалось излучение второй гармоники лазера на красителе Pyrrometene 556 $(\lambda=276.8 \mathrm{~nm}$, ширина линии $\Delta v=380 \mathrm{MHz}$, длительность импульсов по полувысоте $\tau \approx 9 \mathrm{~ns}$ ). На второй ступени перестраиваемое по частоте излучение от второго лазера на красителе Styryl $11(\lambda=773-808 \mathrm{~nm}$, $\tau \approx 13 \mathrm{~ns}, \Delta v \approx 1 \mathrm{GHz})$. В качестве накачки лазеров на красителях применялось излучение лазера на парах меди, работающего с частотой повторения $F=11 \mathrm{kHz}$. Средняя плотность мощности излучения лазера на первой ступени составляла $3 \mathrm{~mW} / \mathrm{cm}^{2}$, а для второй ступени $-1 \mathrm{~W} / \mathrm{cm}^{2}(\lambda=795 \mathrm{~nm})$ в условиях отсутствия насыщения поглощения для этих переходов, приводящего к уширению резонанса поглощения. Коллимированный пучок атомов таллия с концентрацией $\sim 10^{11} \mathrm{~cm}^{-3}$ распространялся между двумя прямоугольными параллельными пластинами из нержавеющей стали размером $6 \times 15 \mathrm{~cm}$ с промежутком между ними $(2 \pm 0.1) \mathrm{cm}$, где пересекался под прямым углом с совмещенными друг с другом излучениями от двух лазеров. Одна пластина заземлялась, а к другой прикладывались регулируемые импульсные или постоянные напряжения. Область взаимодействия определялась поперечным размером лазерного луча в зоне пучка, который представлял собой эллипс с размерами осей $1 \times 2 \mathrm{~cm}$ и длиной $15 \mathrm{~cm}$. Ширина перехода $6 P_{1 / 2} \rightarrow 6 D_{3 / 2}$ в коллимированном атомном пучке таллия на первой ступени существенно сужалось до значения менее $200 \mathrm{MHz}$ по сравнению с шириной допплеровского контура поглощения $\left(\Delta v_{D}=1.7 \mathrm{GHz}\right)$ для атомарного пара, что позволяет разрешить сверхтонкое и изотопическое расщепления основного состояния. Частота излучения лазера второй ступени с линейной поляризацией, параллельной напряженности электрического поля между пластинами, настраивалась на переход $6^{2} D_{3 / 2} \rightarrow n^{2} F_{5 / 2}$, а также могла плавно перестраиваться в широком диапазоне спектра.

Населенность ридберговских состояний (НРС) $n^{2} F_{5 / 2}$ и других, исследуемых в этой работе, может определяться методом полевой ионизации в импульсном электрическом поле, созданном между пластинами после импульсов лазерного возбуждения. При значениях электрического поля, превышающих критическую величину для данного состояния, происходит ионизация атома с эффективностью, близкой к 100\%. По величине электрического тока, возникающего между пластинами, можно определять НРС. Этот метод обладает существенными недостатками в экспериментальных исследованиях эффекта Штарка. Основным из них является неконтролируемое электрическое поле, возникающее из-за наличия остаточного заряда между пластинами от высоковольтного импульса ионизации к моменту $\left(\tau=1 / F \approx 10^{-4} \mathrm{~s}\right)$ прихода следующего лазерного импульса. Поскольку остаточное поле приводит к дополнительным эффектам в спектре возбуждения ридберговских состояний, был использован флуоресцентный метод. В этом случае определение НРС осуществляется путем регистрации сине-зеленой флуоресценции в области спектра $440-460 \mathrm{~nm}$, возникающей за счет дипольноразрешенных распадов $n P_{1 / 2,3 / 2}-7 S_{1 / 2}$ ридберговских состояний атомов Tl. Заселение состояний $n P_{1 / 2,3 / 2}$ после возбуждения состояния $n F_{5 / 2}$ происходит в результате оптических каскадных переходов $n F_{5 / 2} \rightarrow n_{1} D_{3 / 2} \rightarrow n_{2} P_{1 / 2,3 / 2}$. Излучение флуоресценции хорошо регистрируется визуально, а его интенсивность измерялась с помощью ФЭУ в направлении, перпендикулярном распространению атомного пучка и лазерного излучения через отверстие диаметром $1 \mathrm{~cm}$ в центре одной пластины. Апертура отверстия была закрыта проволочной сеткой, чтобы уменьшить искажение электрического поля. Перед входным окном ФЭУ помещались фильтры, отсекающие интенсивное ультрафиолетовое излучение люминесценции и рассеянное излучение накачки на оптических элементах экспериментальной установки. Недостатками данного способа определения НРС является низкая чувствительность регистрации, связанная со свойствами ридберговских состояний. Во-первых, интенсивность флуоресценции быстро падает обратно пропорционально кубу главного квантового числа [12]. Вторая причина связана с большим временем жизни ридберговских состояний, превышающим времена пролета высоковозбужденного атома через наблюдаемую область [12]. Из-за этого отношение сигнал/шум для сигнала с выхода ФЭУ за счет возбужденных состояний $16 F_{5 / 2}$ не превышало 20. Для регистрации спектров 
поглощения применялся box-car-интегратор, аналоговоцифровой преобразователь и компьютер. Одновременно фиксировались частоты излучения перестраиваемых лазеров от измерителя длины волны с погрешностью $300 \mathrm{MHz}$.

\section{Теория}

Энергетическое положение уровня атома таллия $W_{n, L, J}$ с главным квантовым числом $n$, орбитальным моментом $L$ и полным моментом $J$ определяется формулой

$$
W_{n, L, J}=I_{p}-R_{y} / v_{n}^{2}
$$

где $v_{n}=\left(n-\Delta_{L, J}\right)-$ эффективное главное квантовое число $n$-уровня, $I_{p}-$ потенциал ионизации $\left(I_{p}=\right.$ $=49266.55 \mathrm{~cm}^{-1}$ для основного состояния $6^{2} P_{1 / 2}$ изотопа ${ }^{205} \mathrm{Tl}$ с $F=0$ [9]), $R_{y}=109737.024 \mathrm{~cm}^{-1}$ - ридберговская постоянная для атома таллия, $\Delta_{L, J}-$ квантовый дефект. Для расчетов энергии уровней по формуле (1) использовались наиболее точные известные к настоящему времени значения квантовых дефектов $\Delta_{L, J}$. Для состояний $n F_{5 / 2}$ использовались значения $\Delta_{F}=1.0344$ [9], для $n P_{1 / 2}$ и $n P_{3 / 2} \Delta_{P 1}=4.255$ и $\Delta_{P 3}=4.163$ соответственно [13], а для состояний $n S_{1 / 2}$ и $n D_{3 / 2} \Delta_{S}=4.735$ и $\Delta_{D}=3.125[14,15]$.

Во внешнем электрическом поле $E$ сдвиг $\Delta W\left(n, J, m_{J}\right)$ различных магнитных подуровней $m_{J}$ для конкретного электронно-возбужденного состояния может быть выражен через скалярную и тензорную поляризуемости $\alpha_{0}$ и $\alpha_{2}$. Если сверхтонкая структура пренебрежимо мала, что с хорошей точностью выполняется для исследуемых состояний, то штарковский сдвиг и расщепление описывается [16] следующими формулами:

$$
\begin{gathered}
\Delta W\left(n, J, m_{J}\right)=-\frac{E^{2}}{2}\left[\alpha_{0}+\alpha_{2} \frac{3 m_{J}^{2}-J(J+1)}{J(2 J-1)}\right], \\
\alpha_{0}=-\frac{2}{3(2 J+1)} \sum_{k} \frac{\left|\left\langle J|d| J_{k}\right\rangle\right|^{2}}{W_{n}-W_{k}}, \\
\alpha_{2}=2\left[\frac{10 J(2 J-1)}{3(2 J+3)(J+1)(2 J+1)}\right]^{1 / 2} \\
\times \sum_{k} \frac{\left|\left\langle J|d| J_{k}\right\rangle\right|^{2}}{W_{n}-W_{k}}(-1)^{J+J_{k}+1}\left\{\begin{array}{ccc}
J & J_{k} & 1 \\
1 & 2 & J
\end{array}\right\} .
\end{gathered}
$$

Для этих уравнений квадрат матричного элемента $\left|\left\langle J|d| J_{k}\right\rangle\right|^{2}$ для дипольного момента $d=e r$ в $J$-базисе может быть вычислен [16] из квадрата радиального матричного элемента $\left|\left\langle L|d| L_{k}\right\rangle\right|^{2}$ по формуле

$$
\begin{aligned}
\left|\left\langle J|d| J_{k}\right\rangle\right|^{2}= & L_{m}(2 J+1)\left(2 J_{k}+1\right) \\
& \times\left\{\begin{array}{ccc}
L & J & S \\
J_{k} & L_{k} & 1
\end{array}\right\}^{2}\left|\left\langle L|d| L_{k}\right\rangle\right|^{2},
\end{aligned}
$$

где $L_{m}$ - максимальное значение из $L$ и $L_{k}$. Радиальные матричные элементы переходов между ридберговскими состояниями для неводородоподобных атомов в формуле (3) определялись в кулоновском приближении по формулам, представленным в работе [17]:

$$
\left\langle L|d| L_{k}\right\rangle=\left\{\frac{3}{2} v_{c}^{2}\left[1-\left(L_{m} / v_{c}\right)^{2}\right]^{1 / 2}\right\} \sum_{p=0}^{3} \gamma^{p} g_{p}(s),
$$

где $v_{c}=2 /\left(1 / v_{n}+1 / v_{k}\right), \quad s=v_{n}-v_{k}, \quad \gamma=\Delta L\left(L_{m} / v_{c}\right)$, $\Delta L=L_{k}-L$. Значения для функций $g_{p}(s)$ приведены в работе [17]. В работе [18] утверждается, что этот метод расчета радиальных матричных элементов дает погрешность менее $0.1 \%$ при больших значениях $v_{n}$ и $v_{k}$. Следует отметить, что основной вклад в расчетах поляризуемостей ридберговских состояний дают соседние близлежащие переходы с $s \ll 1$. Состояния с $s \geq 1$ в формуле (4) для дипольных моментов перехода дают значения на три порядка меньшие и соответственно продолжают быстро уменьшаться с увеличением $s$ и $\Delta L$. Для ридберговских состояний скалярная и тензорная поляризации с ростом главного квантового числа растут как $v^{7}[12]$. Отсюда следует, что вклад в поляризуемость нижнего сильно связанного состояния $3 D_{3 / 2}$ в расчетах можно не учитывать, поскольку он значительно меньше, чем для рассматриваемых верхних ридберговских состояний. Штарковская структура в неводородоподобных атомах даже для простейших атомов щелочных металлов является слишком сложной, чтобы полностью описать ее набором параметров. В большинстве случаев ограничиваются построением диаграммы энергии уровней в зависимости от напряженности электрического поля.

Во внешнем даже слабом электрическом поле полный момент $J$ перестает сохраняться. В однородном поле сохраняется лишь проекция момента $m_{J}$. Поэтому при радиационных переходах даже в слабом поле правила отбора по $J$ не обязательны, и в спектре атомов появляются дипольнозапрещенные линии. Такие запрещенные переходы, индуцированные электрическим полем, регистрировались экспериментально и ранее, начиная с открытия эффекта Штарка. Вычисление интенсивностей этих линий сводится к вычислению поправок к волновым функциям стационарных состояний атомов $[19,20]$. Количественные измерения для интенсивности запрещенных переходов между низколежащими состояниями атомов цезия и сравнение этих результатов с расчетом сообщались в работе [20]. Подобные эксперименты были проведены в работе [21] с использованием более сложной системы, состоящей из большого числа штарковских компонент в узком диапазоне энергий для ридберговских состояний атома ксенона. В ридберговских атомах таллия с большими значениями квантовых дефектов для состояний с высокими значениями орбитальных моментов может возникать ряд новых особенностей в поведении энергии уровней в электрическом поле. 


\section{Результаты экспериментов и обсуждение}

В отсутствие статического электрического поля в области взаимодействия пучка атомов таллия с лазерным излучением фиксировались только оптические переходы на состояния $16 F_{5 / 2}$ с шириной резонанса на второй ступени $1.1 \mathrm{GHz}$ (кривая 1 на рис. 1). В то же время экспериментально не были зарегистрированы дипольноразрешенные переходы на соседние состояния $19 P_{1 / 2}$ и $19 P_{3 / 2}$. Силы осцилляторов $f$ этих переходов рассчитывались в приближении Бэйтса-Дамгаарда [22] с помощью таблиц, приведенных в [23]. Для перехода $6 D_{3 / 2} \rightarrow 16 F_{5 / 2}$ расчетное значение равно $f=1.5 \cdot 10^{-3}$. Для других оптических переходов $6 D_{3 / 2} \rightarrow 19 P_{1 / 2}$ и $6 D_{3 / 2} \rightarrow 19 P_{3 / 2}$ (состояния $19 P$ расположены по энергии близко с состоянием $16 F_{5 / 2}$ ) силы осцилляторов оказались соответственно в 50 и 3000 раз меньше. Отсюда следует, что вероятности этих переходов малы, и поэтому они не наблюдаются в эксперименте при отношении сигнал/ шум $\sim 20$ для интенсивной флуоресценции с возбужденных состояний $16 F_{5 / 2}$.

При включении электрического поля центр резонанса $6 D_{3 / 2}-16 F_{5 / 2}$ смещается в длинноволновую сторону, и ширина его увеличивается (кривая 2 на рис. 1). На рис. 2 представлена картина энергетического положения центров оптических переходов из $6 D_{3 / 2}$ в ридберговские состояния атома ${ }^{205} \mathrm{Tl}$, зарегистрированных вблизи уровня $16 F_{5 / 2}$, в зависимости от приложенного электрического поля. Значение нулевой энергии на оси ординат совпадает с положением энергии $E=48776.57 \mathrm{~cm}^{-1}$ для уровня $16^{2} F_{5 / 2}(F=2.3)$, которое отсчитывается от самого нижнего уровня $6^{2} P_{1 / 2}^{205} \mathrm{Tl}(F=0)$. Энергетическое положе-

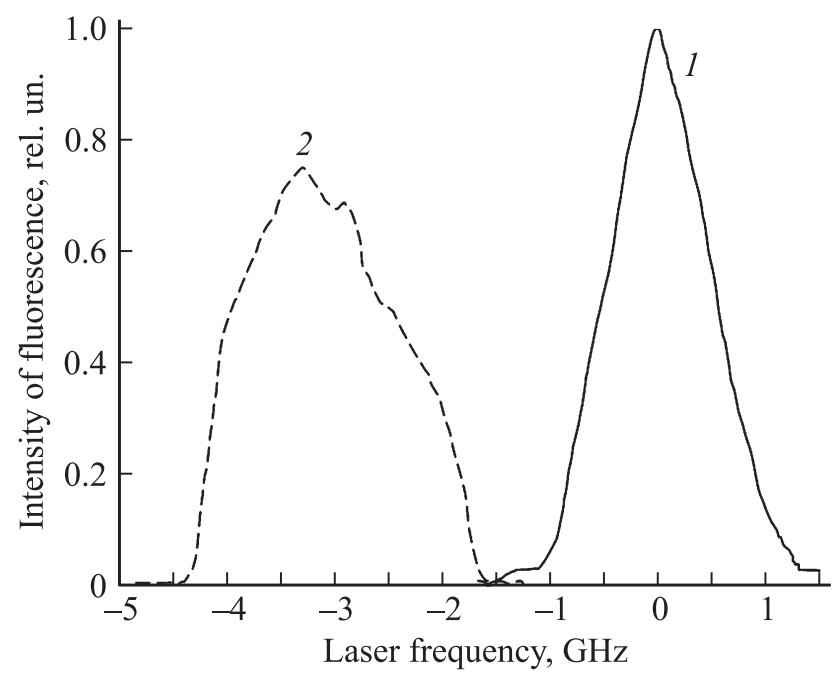

Рис. 1. Спектры изотопически селективного возбуждения коллимированного пучка атомов ${ }^{205} \mathrm{Tl}$ на оптическом переходе второй ступени $6^{2} D_{3 / 2} \rightarrow 16^{2} F_{5 / 2}$ для двух значений напряженности приложенного электрического поля: $E=0$ (1), $0.2 \mathrm{kV} / \mathrm{cm}(2)$. ние исследуемых в работе состояний и значения тонкого расщепления взяты из работ $[9,13-15]$. Значение тонкого расщепления для $F$-состояний в атоме Т1 неизвестно. Расчеты по формуле из [24] для уровней $16 F_{5 / 2}$ и $16 F_{7 / 2}$ при сравнении с тонким расщеплением для $D$-состояний показывают, что оно меньше, чем $0.36 \mathrm{~cm}^{-1}$. Также неизвестны значения квантовых дефектов и тонкой структуры для состояний $16 G_{7 / 2,9 / 2}$. При напряженности электрического поля $E \leq 1 \mathrm{kV} / \mathrm{cm}$ энергетические смещения уровней имеют значения, меньшие, чем расстояния между уровнями, и поэтому их можно описывать формулами как для квадратичного эффекта Штарка. Огромные значения квантовых дефектов энергетических уровней в атоме таллия, вызванные сложным строением атомного ядра, приводят к неводородоподобному поведению штарковской структуры вплоть до напряженностей электрического поля $1.5 \mathrm{kV} / \mathrm{cm}$. Этим таллий значительно отличается от атомов щелочных металлов [6]. Экспериментальные результаты (кривая 2 на рис. 1) показывают, что расщепленные компоненты в регистрируемом спектре практически сливаются. Это связано c конечной спектральной шириной линии излучения лазера на второй ступени и наличием неоднородности в приложенном электрическом поле, которые приводят к дополнительному уширению расщепленных спектральных компонент $m_{J}$.

Поскольку в работе использовался лазер на второй ступени возбуждения с линейной поляризацией, параллельной напряженности приложенного электрического поля, то согласно правилам отбора для магнитного квантового числа, $\Delta m=0$, из состояния $6 D_{3 / 2}$ возбуждаются только уровни энергии с $m_{J}=1 / 2,3 / 2$ состояний $16^{2} F_{5 / 2}, 18^{2} D_{3 / 2}$ и $16^{2} G_{7 / 2}$.

Рис. 2 показывает, что в окрестности состояния $16 F_{5 / 2}$ наряду с переходом $6 D_{3 / 2} \rightarrow 16 F_{5 / 2}$ с частотой $v_{1}$ в электрическом поле появляются линии поглощения на дипольнозапрещенных переходах $6 D_{3 / 2} \rightarrow 18 D_{3 / 2,5 / 2}$ с частотой $v_{2}$. Это связано с перемешиванием состояний $16 F$ и $18 D$, вызванным электрическим полем. Соответственно в спектре поглощения присутствуют две линии с частотами $v_{1}$ и $v_{2}$, принадлежащие соответственно переходам $6 D_{3 / 2} \rightarrow 16 F_{5 / 2}$ и $6 D_{3 / 2} \rightarrow 18 D_{3 / 2}$. В экспериментальном спектре количество этих запрещенных линий больше, поскольку в оптических переходах участвуют большое число близкорасположенных состояний, в том числе компоненты тонкой структуры. В слабом электрическом поле, когда дипольный момент перехода $|\langle 16 F|d| 18 D\rangle| \ll h\left(v_{1}-v_{2}\right)$, интенсивность линии $v_{1}$ практически не зависит от напряженности поля, а интенсивность $I_{2}$ линии поглощения с частотой $v_{2}$ пропорциональна квадрату напряженности поля [19]:

$$
I_{2}=C|\langle 16 F|d| 18 D\rangle|^{2} /\left[h\left(v_{1}-v_{2}\right)\right]^{2},
$$

где $C$ - постоянная. В сильном электрическом поле, когда $|\langle 16 F|d| 18 D\rangle| \gg h\left(v_{1}-v_{2}\right)$, интенсивности линий $v_{1}$ и $v_{2}$ становятся равными. Правилами отбора 


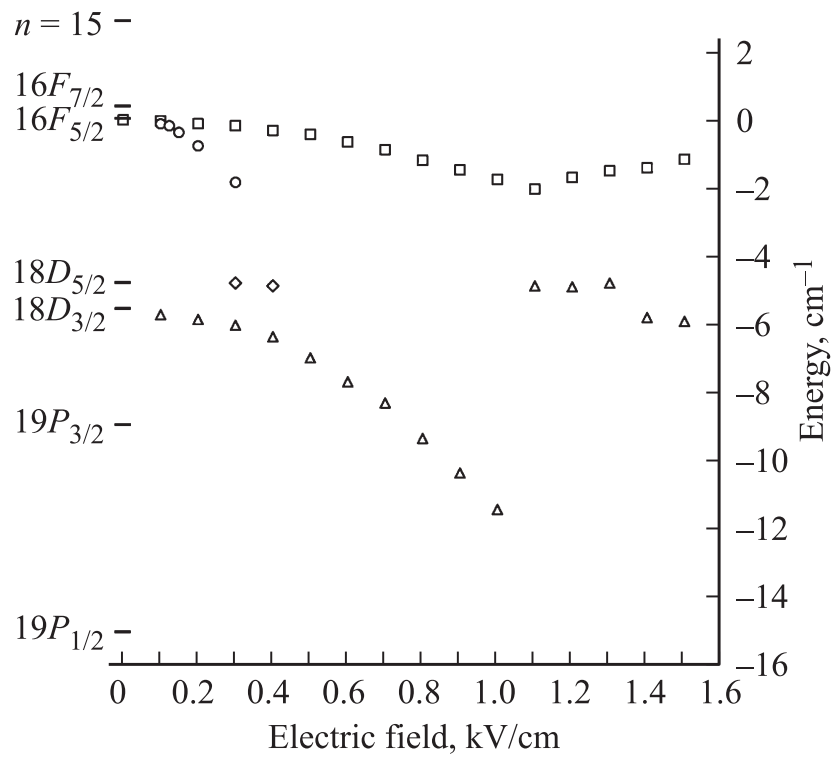

Рис. 2. Энергетическое положение центров оптических переходов из состояния $6 D_{3 / 2}$ в ридберговские состояния атома ${ }^{205} \mathrm{Tl}$ в зависимости от напряженности приложенного электрического поля. Значение нулевой энергии на оси ординат совпадает с положением энергии $E=48776.57 \mathrm{~cm}^{-1}$ для уровня $16^{2} F_{5 / 2}(F=2.3)$, которое отсчитывается от самого нижнего уровня $6^{2} P_{1 / 2}{ }^{205} \mathrm{Tl}(F=0)$.

для матричного элемента из формулы (5) в присутствии электрического поля разрешены два запрещенных перехода $6 D_{3 / 2}-18 D_{3 / 2}$ и $6 D_{3 / 2}-18 D_{5 / 2}$ (тонкое расщепление $0.76 \mathrm{~cm}^{-1}$ ). Однако расчеты по формулам (3)-(5) показывают, что интенсивность второго перехода в 14 раз меньше интенсивности первого, т.е. его вклад в наблюдаемую картину спектра мал. Также в присутствии электрического поля экспериментально наблюдаются запрещенные переходы на штарковские компоненты $m_{J}=1 / 2,3 / 2$ для состояния $16 G_{7 / 2}$, расположенного выше состояния $16 F_{5 / 2}$. Поэтому их необходимо учитывать при расчете скалярной поляризации для состояния $16 F_{5 / 2}$.

При изучении оптических переходов, индуцированных электрическим полем, в некоторых случаях может возникнуть неоднозначность в интерпретации результатов экспериментов. Как ранее отмечалось, что в присутствии внешнего, даже слабого электрического поля можно оперировать корректно лишь величиной проекции момента $m_{J}$ для конкретного энергетического состояния атома. Представим, что после возбуждения некоторой штарковской компоненты $m_{J}$ происходит выключение электрического поля. В каком энергетическом состоянии оказывается в этом случае свободный от электрического поля атом? На примере атомов натрия в работе [25] производился анализ свободных от поля ридберговских состояний атомов методом миллиметровой спектроскопии после возбуждения штарковских компонент $m_{J}$ и выключения электрического поля. Во всех случаях возбуждения различных штарковских компонент, в том числе для запрещенных переходов, атомы после выключения поля оказывались только в определенных энергетических состояниях, которые соответствуют дипольноразрешенным оптическим переходам из начального состояния. Следовательно, находящийся в электрическом поле возбужденный атом при выключении поля может переходить (релаксировать) только в такие состояния, которые связаны с начальным через дипольноразрешенные переходы.

Рис. 3 представляет результаты экспериментов для положения энергии уровней и значения амплитуд сигналов флуоресценции в зависимости от приложенного электрического поля. Кривые 1, 2 и 3 отображают штарковский сдвиг состояний $16 F_{5 / 2}, 16 G_{7 / 2}$ и $18 D_{3 / 2}$ соответственно. Кривые 4 и 5 демонстрируют относительные амплитуды сигналов флуоресценции, возникающих изза распада состояний $16 F_{5 / 2}$ и $18 D_{3 / 2}$ соответственно. Амплитуда сигнала флуоресценции (кривая 4) от распада $16 F_{5 / 2}$ монотонно падает с усилением приложенного электрического поля, хотя по теории, представленной в [19], она не должна уменьшаться в некотором начальном интервале напряженности электрического поля. Наиболее вероятная причина спада сигнала связана с уширением линии поглощения на оптическом переходе $6 D_{3 / 2} \rightarrow 16 F_{5 / 2}$ за счет штарковского расщепления, а вследствие этого уширения верхнего состояния при монохроматическом возбуждении излучением лазера на второй ступени. Напротив, амплитуда сигнала (кривая 5) от запрещенного перехода $6 D_{3 / 2} \rightarrow 18 D_{3 / 2}$ сначала растет при увеличении напряженности электрического поля, а затем падает. Начальный рост амплитуды сигнала близок к квадратичной зависимости, которая следует из

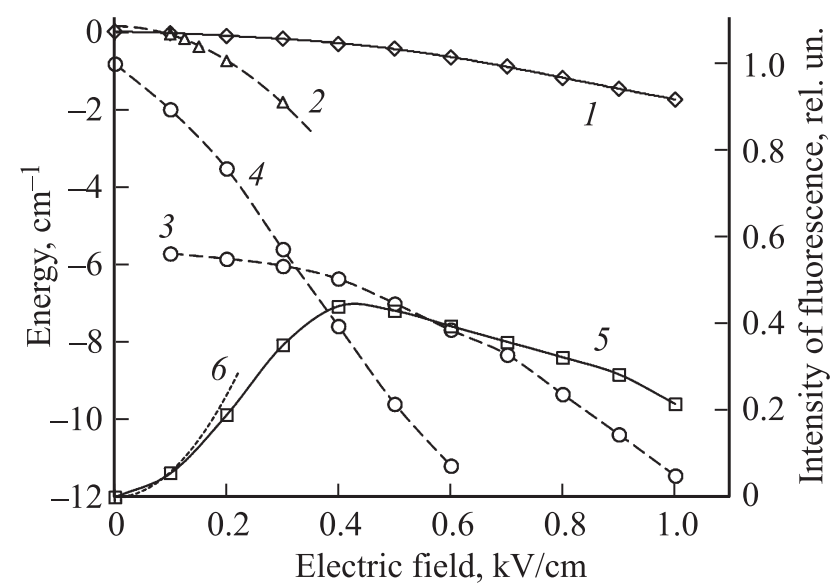

Рис. 3. Смещение энергии уровней и значения амплитуд сигналов флуоресценции для ${ }^{205} \mathrm{Tl}$ в окрестности состояния $16 F_{5 / 2}$ в зависимости от напряженности приложенного электрического поля: $1-3-$ штарковский сдвиг состояний $16 F_{5 / 2}$, $16 G_{7 / 2}$ и $18 D_{3 / 2}$ соответственно; 4,5 - амплитуды сигналов флуоресценции, возникающих из-за распада состояний $16 F_{5 / 2}$ и $18 D_{3 / 2}$ соответственно; $6-$ аппроксимация начального участка кривой 5 по формуле (3). 


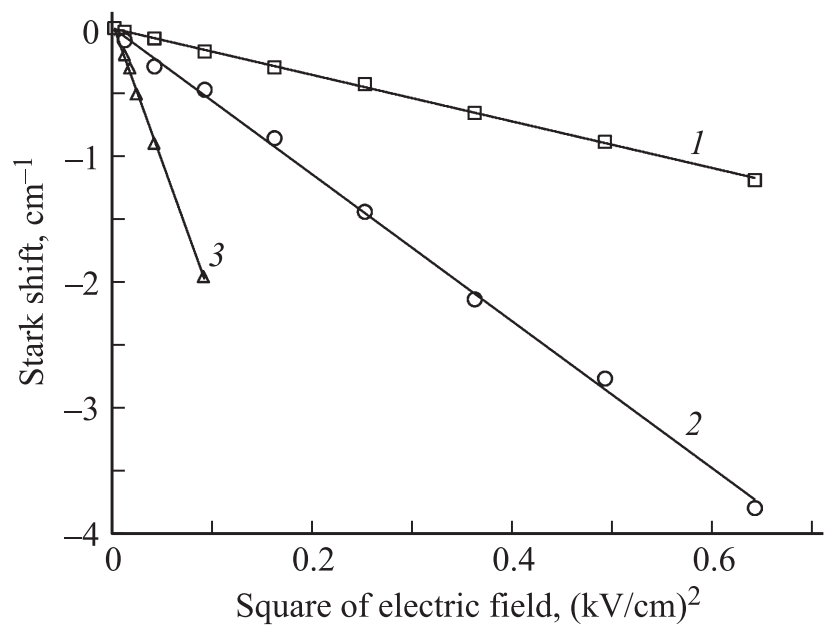

Рис. 4. Зависимости сдвига энергии уровней от квадрата напряженности электрического поля. 1 - экспериментальные данные для состояния $16^{2} F_{5 / 2}$ и их аппроксимация методом наименьших квадратов по формуле (2); 2,3 - то же для состояний $18^{2} D_{3 / 2}$ и $18^{2} G_{3 / 2}$ соответственно.

формулы (5) и которая иллюстрируется кривой 6 на рис. 3. Спад обусловлен той же причиной, что и для кривой 4.

Рис. 4 представляет экспериментальные зависимости штарковского сдвига энергии уровней от квадрата напряженности приложенного электрического поля $E^{2}$ для состояний $16^{2} F_{5 / 2}, \quad 18^{2} D_{3 / 2}$ и $16^{2} G_{7 / 2}$ и их аппроксимацию методом наименьших квадратов по формуле (2). Отсюда получены экспериментальные значения для скалярных поляризуемостей (в ед. $\left.\mathrm{cm}^{-1} /(\mathrm{kV} / \mathrm{cm})^{2}\right): \alpha_{0}\left(16 F_{5 / 2}\right)=3.71 \pm 0.3, \alpha_{0}\left(18 D_{3 / 2}\right)=$ $=11.7 \pm 0.25$ и $\alpha_{0}\left(16 G_{7 / 2}\right)=44.1 \pm 0.9$.

Для расчетов скалярной и тензорной поляризуемостей для состояний $18 D_{3 / 2}$ использовались известные данные и результаты наших измерений. В этом случае принимаются в расчет только три соседние состояния $16 F_{5 / 2}$, $19 P_{3 / 2}$ и $19 P_{1 / 2}$ (рис. 2). Вклад других уровней, как показал анализ, мал по сравнению с погрешностью измерения. Расчет радиальных матричных элементов по формуле (4) для переходов $18 D_{3 / 2}-16 F_{5 / 2}, 18 D_{3 / 2}-19 P_{3 / 2}$ и $18 D_{3 / 2}-19 P_{1 / 2}$ показал значения $\left\langle L|d| L_{k}\right\rangle=328.6,326.9$ и 292.8 a.u. соответственно. Значения энергии уровня $W_{0}$ и квантового дефекта $\Delta_{D}$ для состояния $18 D_{3 / 2}$ сообщали ранее в работах $[14,15]$. В настоящей работе получены более чем на порядок точные значения путем аппроксимации экспериментальных данных на рис. 3 методом наименьших квадратов по формуле $W_{18 D}=W_{0}-b E^{2}$. Полученные значения составляют $W_{0}=-(5.56 \pm 0.04) \mathrm{cm}^{-1}, \Delta_{D}=3.1184$. Достигнутая точность измерений реализована за счет использования более узких резонансов поглощения и повышения точности измерений частоты излучения лазеров. Значения отстроек частоты $\left(W_{n}-W_{k}\right)$ в формуле (2) для состояний $18 D_{3 / 2}$ и $19 P_{3 / 2}, 19 P_{1 / 2}$, равные 3.43 и $9.52 \mathrm{~cm}^{-1}$ соответственно, заимствованы из данных $[13,14]$ и не превышают ошибку их измерения $<1 \mathrm{~cm}^{-1}$. Расчетное значение для скалярной поляризуемости по формуле (2) равно $\alpha_{0}\left(18 D_{3 / 2}\right)=6.65 \mathrm{~cm}^{-1} /(\mathrm{kV} / \mathrm{cm})^{2}$. Расхождение между теорией и экспериментом составляет $\sim 43 \%$, что является предметом дальнейшего уточнения теоретической модели, поскольку все исходные параметры определены с большой точностью. Этого следовало ожидать для сложной системы, состоящей из нескольких близкорасположенных взаимодействующих уровней. Расчетная модель не учитывает, например, влияние тонкого расщепления состояния $18 D_{3 / 2}$, которое сравнимо со штарковским сдвигом уровней. Можно ожидать, что уточненная теоретическая модель позволит уменьшить различие расчетных значений с экспериментальными. Расчет тензорной поляризуемости показал, что ее значение составляет менее $5 \%$ от скалярной поляризуемости. Верхний уровень $18 D_{3 / 2}$ расщепляется только на две симметричные компоненты, которые одновременно возбуждаются, а поэтому не вносят ошибку в измерение скалярной поляризуемости.

При расчетах скалярной и тензорной поляризуемостей уровня $16 F_{5 / 2}$ по формуле (2) достаточно учесть только соседние состояния $18 D_{3 / 2}$ и $16 G_{7 / 2}$. Энергия уровня $18 D_{3 / 2}$ и его отстройка от состояния $16 F_{5 / 2}$ известны с хорошей точностью. Однако остаются неизвестными положения энергии $G$-состояний атома таллия. Поэтому было определено значение энергии для состояния $16 G_{7 / 2}$ из предположения, что расчетная и измеренная величины для поляризуемости совпадают. В этом случае наилучшее согласие между этими величинами достигает при отстройке уровня $16 G_{7 / 2}$ от $16 F_{5 / 2}$, равной $5.41 \mathrm{~cm}^{-1}$. Этой величине соответствуют значения радиального матричного элемента для перехода $16 F_{5 / 2}-16 G_{7 / 2}\left\langle L|d| L_{k}\right\rangle=320.16$ a.u. и квантового дефекта состояния $16 G_{7 / 2} \Delta_{G}=0.95$. Верхний уровень $16 F_{5 / 2}$ расщепляется на три компоненты с $m_{J}=5 / 2,3 / 2,1 / 2$, но только две последние возбуждаются согласно правилам отбора для магнитного квантового числа $\Delta m=0$ из состояния $6 D_{3 / 2}$. Вследствие этого расщепления центр тяжести суммарной линии поглощения для состояния $16 F_{5 / 2}$ смещается вниз по энергии на величину, не превышающую $10 \%$. Это смещение не превышает погрешности измерения скалярной поляризуемости состояния $16 F_{5 / 2}$. Поэтому расщепление уровня $16 F_{5 / 2}$, вызванное присутствием электрического поля, не оказывает заметного влияния на точность измерения его скалярной поляризуемости.

\section{Заключение}

Эффект Штарка для ридберговских состояний атомов таллия экспериментально исследован в окрестности $16^{2} F_{5 / 2}$-уровня с использованием коллимированного атомного пучка с двухступенчатым изотопически селективным возбуждением. Основные атомные данные, 
такие как электрические поляризуемости и энергетические параметры $16 F_{5 / 2^{-}}, 162 G_{7 / 2^{-}}$и $18 D_{3 / 2}$-состояний, были определены впервые. Большая плотность энергетических состояний в малом спектральном интервале из-за больших квантовых дефектов и тонкой структуры таллия приводит к спектрам, которые довольно сложно описать теоретически. Тем не менее изучены особенности спектров, а также проведены сравнения с расчетами в рамках простой модели. Экспериментально наблюдалось сильное перемешивание энергетических состояний в электрическом поле, имеющих различную четность. Это позволяет эффективно возбуждать атомы таллия с большой концентрацией через запрещенные переходы, подстраивая частоту излучения перехода на частоту возбуждающего излучения с помощью слабого электрического поля.

\section{Список литературы}

[1] Porsev S.G., Safronova M.S., Kozlov M.G. // Phys. Rev. Lett. 2012. V. 108. P. 173001.

[2] Mitroy J., Safronova M.S., Clark C.W. // J. Phys. B. 2010. V. 43. P. 202001.

[3] Rochester S., Bowers C.J., Budker D., DeMille D., Zolotorev M. // Phys. Rev. A. 1999. V. 59. P. 3480.

[4] Vetter P.A., Meekhof D.M., Majumder P.K., Lamoreaux S.K., Fortson E.N. // Phys. Rev. Lett. 1995. V. 74. P. 2658.

[5] Safronova M.S., Mitroy J., Clark C.W., Kozlov M.G. // AIP Conf. Proc. 2015. V. 1642. P. 81.

[6] Zimmerman M.L., Littman M.G., Kash M.M., Kleppner D. // Phys. Rev. A. 1979. V. 20. P. 2251.

[7] Dong H.J., Wang X.Y., Li C.Y., Jia S.T. // Acta Phys. Sin. 2015. V. 64. P. 093201.

[8] Xu L., Zhao Y., Zhang M., He M., Wang G., Wang Z. // Opt. Commun. 1991. V. 82. P. 12.

[9] Бохан П.А., Закревский Дм.Э., Ким В.А., Фатеев Н.В. // Опт. и спектр. 2008. Т. 104. № 4. С. 598.

[10] Bokhan P.A., Zakrevsky Dm.E., Kim V.A., Fateev N.V. // Laser Phys. 2009. V. 19. P. 2050.

[11] Bokhan P.A., Zakrevsky Dm.E., Kim V.A., Fateev N.V. // Laser Phys. 2013. V. 23. P. 055702.

[12] Stebbings R.F., Dunning F.B. Rydberg States of Atoms and Molecules. Cambridge: Cambridge University Press, 1982. 496 р. Перевод: Стеббингс Р., Даннинг Ф. Ридберговские состояния атомов и молекул. М.: Мир, 1985. 495 с.

[13] Riaz M., Mahmood S., Anwar-ul-Haq M., Baig M.A. // Opt. Commun. 2005. V. 244. P. 339.

[14] Riaz M., Anwar-ul-Haq M., Raheel A., Baig M.A. // Opt. Commun. 2010. V. 2283. P. 5046.

[15] Алимов У.Ж., Мухдабаев И.Ш., Тухлебаев О., Турсунов А.T. // Опт. и спектр. 2001. Т. 90. № 6. С. 899.

[16] Hunter L.R., Krause D., Murthy Jr.S., Sung T.W. // Phys. Rev. 1988. V. 37. P. 3283.

[17] Edmondst A.R., Picartt J., Minht N.T., Pullen R. // J. Phys. B. 1979. V. 12. P. 2781.

[18] Knight R.D., Wang L. // Phys. Rev. A. 1985. V. 32. P. 896.

[19] Бонч-Бруевич А.М., Ходовой В.А. // УФН. 1967. Т. 93. C. 71.

[20] Zimmermann P., Ducas T.W., Littman M.G., Kleppner D. // Opt. Commun. 1974. V. 12. P. 198.
[21] Knight R.D., Wang L. // Phys. Rev. A. 1985. V. 32. P. 2751.

[22] Bates D.R., Damgard A. // Phil. Trans. Roy. Soc. London. 1949. V. 242. Р. 101.

[23] Собельман И.И. Введение в теорию атомных спектров. М.: Наука, 1977. С. 319.

[24] Riaz M., Anwar-ul-Haq M., Raheel A., Baig M.A. // Opt. Commun. 2004. V. 233. P. 323.

[25] Фатеев Н.В. // Опт. и спектр. 1994. Т. 76. № 4. С. 558. 